\title{
La colección de peinetas del Museo del Traje, CIPE. Problemática de conservación
}

\author{
Elena Gómez Sánchez, María Antonia Herradón Figueroa, Silvia Montero Redondo y \\ María Antonia García
}

\begin{abstract}
Resumen: En la colección de peinetas del Museo del Traje. Centro de Investigación del Patrimonio Etnológico (Madrid) figuran ejemplares de los siglos XVIII al XX, un espectro temporal muy amplio que se corresponde con una gran diversidad de materiales de fabricación. Entre ellos destaca el nitrato de celulosa (NC), el más importante de los primeros polímeros producido a escala industrial. El proceso de degradación de este material está relacionado, entre otros fenómenos, con la pérdida del plastificante utilizado para su manufactura, el alcanfor, pérdida que puede seguirse por FTIR. Dicha degradación es autocatalítica e irreversible, y depende en gran medida de las condiciones ambientales. A pesar de su inestabilidad, un adecuado control de temperatura y humedad, entre otros parámetros, permite alargar considerablemente la vida de estos materiales, cuya vigilancia es imprescindible dado, además, el carácter propagativo de los procesos de degradación de unas piezas a otras.
\end{abstract}

Palabras clave: peinetas, nitrato de celulosa, alcanfor, FTIR, Mylar, conservación preventiva, restauración.

Abstract: The hair combs collection of the Museo del Traje. Centro de Investigación del Patrimonio Etnológico (Madrid) contains objects from the $18^{\text {th }}-20^{\text {th }}$ centuries. This broad time span allows a wide range of materials to be found as components. Among them is one of the first and most important semi-synthetic polymers to be manufactured, cellulose nitrate (NC). A noticeable phenomenon of the degradation process of this material is the loss of camphor, used as plasticizer and stabiliser, event that can be followed by FTIR. The degradation of this material is autocatalytic and permanent, and is highly dependent on ambient conditions. An adequate control of the conservation environment and a close vigilance of the objects can increase the life of these materials.

Keywords: hair combs, cellulose nitrate, camphor, FTIR, Mylar, preventive conservation, restauration.

Resumo: Na colecção de peinetas do Museo del Traje, Centro de Investigación del Patrimonio Etnológico (Madrid) figuram exemplares dos séculos XVIII ao XX, um espectro temporal muito amplo que se corresponde com uma grande diversidade de materiais de fabricação. Entre eles destaca-se o nitrato de celulose (NC): o mais importante dos primeiros polímeros produzido à escala industrial. O processo de degradação de este material está relacionado, entre outros fenómenos, com a perda do plastificante utilizado para a sua manufactura, o alcanfor, perda que pode seguir-se por FTIR. Dita degradação é auto-catalítica e irreversível, e depende em grande medida das condições ambientais. Apesar da sua inestabilidade, um adequado controlo da temperatura e humidade, entre outros parámetros, permite alongar considerávelmente a vida destes materiais, cuja vigilância é imprescindible, tendo em conta o carácter propagativo dos processos de degradação de umas peças a outras.

Palavras-chave: peinetas, nitrato de celulose, alcanfor, FTIR, Mylar, conservação preventiva, restauração.

\section{Introducción}

\section{La colección de peinetas del Museo del Traje}

Entre las numerosas colecciones que configuran el actual Museo del Traje. Centro de Investigación del Patrimonio Etnológico (Madrid) vamos a detenernos aquí en la formada por peinetas y peinecillos, unos accesorios íntimamente ligados tanto a la historia del vestido en general como a la particular trayectoria de la indumentaria española. Para empezar diremos que, en esencia, peineta y 
peinecillo deben considerarse sinónimos a los efectos que nos van a ocupar, ya que uno y otro vocablo sólo se hacen eco de matices relativos al tamaño de la pieza en cuestión o a la importancia de la decoración que ostenta la crestería. Ambos términos se refieren, en definitiva, a una especie de peine, utilizado en culturas muy diversas a lo largo del tiempo, en el que confluyen dos funciones: una utilitaria (sujeción del cabello, soporte de textiles) y otra ornamental (adorno de la cabeza). Así pues, como en la práctica se trata de una sinonimia, para referirnos a tales objetos hablaremos de peinetas.

A pesar de su universalidad, hace aproximadamente un siglo las peinetas se convirtieron, merced a diversas circunstancias de índole social y política, en una seña indefectible de la idiosincrasia española. Esta circunstancia motivó su temprana presencia en las colecciones del Museo del Pueblo Español, una institución que nació en 1934 con el objetivo de reunir y preservar todos los objetos relacionados con nuestra cultura popular. $\mathrm{Y}$ aunque desde entonces han sido varias las orientaciones científicas que ha tenido el centro, lo cierto es que la colección ha venido experimentado un crecimiento constante hasta llegar a las casi 150 piezas que la componen en la actualidad.

Una particularidad de la colección que nos ocupa es que, salvo contadísimas excepciones y siempre durante períodos de tiempo muy breves, nunca se ha expuesto al público. De ahí que a partir de 1986 se prestara especial atención a su ubicación en las salas de reserva del Museo, un espacio diseñado tanto para que las piezas gozaran de las mejores condiciones posibles de conservación como para que los investigadores pudieran acceder a ellas con comodidad. Así, al igual que todos los objetos de pequeño tamaño, en los últimos veinte años las peinetas se han venido guardando en archivadores metálicos, en cajones provistos de bandejas de madera sin tratar, recubiertas a su vez con una base textil neutra. El sistema ha permanecido invariable desde entonces, algo que no ha ocurrido en el caso del envoltorio usado como protector individual de cada peineta, el cual puede ser bien de un polímero sintético (polietileno y tereftalato de polietileno) bien de un material celulósico.

A comienzos de la década de 1990 el Museo estableció para las salas de reserva un protocolo de actuación que incluía la revisión periódica de las colecciones por parte del conservador responsable de cada una de ellas. En el caso de las peinetas, las revisiones efectuadas en los últimos quince años nos han servido para realizar el seguimiento de su estado de conservación en cada momento, constatar su progresivo deterioro y poner de manifiesto la necesidad de efectuar análisis químicos para conocer con exactitud las características de los materiales de fabricación. En la actualidad, a la vista de los resultados obtenidos y en colaboración con el departamento de conservación del Museo, hemos conseguido establecer un programa de actuación en materia de conservación y restauración para este tipo de colección.

\section{Materiales de fabricación}

En la fabricación de peinetas se han venido utilizando históricamente dos tipos de materias. Por un lado, metales y aleaciones como el oro, la plata, el latón y el acero; por otro, materiales de origen natural como el hueso, el marfil y el carey. Todos ellos tienen en común un alto precio, derivado de su escasez y de la complejidad técnica de su manufactura, y unas limitaciones formales, derivadas del peso, color o dimensiones de la materia en cuestión. A partir de la segunda mitad del siglo XIX, el desarrollo industrial permitió superar estas barreras gracias a la invención de los primeros polímeros sintéticos. Así pues, lo que comúnmente denominamos "plástico" permitió satisfacer la creciente demanda de todo tipo de objetos utilizados en la vida cotidiana, entre ellos las peinetas, gracias a su moderado precio, a su casi perfecta imitación de materias nobles y, sobre todo, a sus extraordinarias posibilidades decorativas. Si antes del desarrollo de la gran familia de los plásticos para elaborar una peineta de las llamadas españolas, caracterizada por su gran tamaño, se necesitaba la concha completa de una tortuga y la maestría de un peinetero, a partir de ahí el uso de moldes simplificó el proceso e incrementó la producción. 
Desafortunadamente, como muestran las piezas que presentamos aquí, los materiales poliméricos no sólo presentan ventajas. También sufren procesos de deterioro debido en buena medida a su propia naturaleza, por lo que parece imprescindible conocer las características de sus componentes y su comportamiento. Se trata en definitiva de una problemática muy frecuente en determinados museos que por fortuna en los últimos años ha empezado a ser abordada mediante el concurso de equipos de trabajo interdisciplinares (Espinoza Monasterio y Araya Monasterio 2006).

Entre los materiales poliméricos dos de los más vulnerables son el nitrato de celulosa (NC) y el acetato de celulosa (AC). La mayoría de los objetos fabricados en nitrato de celulosa data de los años 1846-1950 (Reilly 1991: 145-162), habiendo comenzado su manufactura comercial en el año 1866 y marcando así el comienzo del desarrollo industrial de los plásticos. Por su influencia en el desarrollo de las artes y la tecnología ha sido considerado un modelo a la vez positivo y negativo, por adelantar algunas de las ventajosas propiedades que los plásticos llegarían a tener con el tiempo, en el primer caso, y por ejemplarizar algunos de los inconvenientes de su uso que ocuparían a la industria química durante el siglo XX, en el segundo.

Este polímero de origen semisintético, obtenido por nitración de la celulosa, conserva desde el punto de vista químico la estructura básica de la cadena en la que algunos grupos funcionales han sido transformados en nitratos. La fragilidad del NC como tal, sin embargo, hizo en primera instancia impracticable su utilidad para fabricar objetos que pudieran ser usados de forma cotidiana. (Albus et al. 2007: 23). La solución a este problema vino de la mano de Alexander Parkes, que introdujo la idea de añadir aceites vegetales para disminuir dicha fragilidad, idea que fue finalmente llevada a cabo con éxito por John Wesley Hyatt, quien acertadamente eligió el alcanfor como aditivo (plastificante) para este propósito, siendo así capaz de conseguir la maleabilidad deseada. La moléculas de este compuesto, mucho menores que las cadenas del NC, son capaces de intercalarse entre éstas últimas, permitiendo así cierto movimiento entre ellas y dotando al producto resultante de la plasticidad (Albus et al. 2007: 76) necesaria para moldearlo.

Así, con la adición de alcanfor como plastificante (Selwitz 1988), el NC fue utilizado para fabricar imitaciones de materiales naturales preciosos, escasos y muy cotizados, como marfil, carey o cuerno, para lo que era muy apreciado dado su gran parecido y su agradable tacto. La limitada disposición de estos productos en la naturaleza condicionaba tanto su disponibilidad en el mercado como su elevado precio, mientras que el nitrato de celulosa empleado como imitación de estos materiales era de obtención fácil y abarataba la fabricación de los accesorios mencionados.

Con el cambio de siglo, las extraordinarias posibilidades de este polímero fomentaron su desarrollo en otros ámbitos, y así surgió su utilización como material fotográfico y fílmico en 1889 y 1895 respectivamente (Lavédrine 2003). Una vez que su aparición revolucionó el desarrollo de la fotografía, las propiedades de este material hicieron técnicamente posible su extensión al nacimiento de la cinematografía. Con el tiempo, este material sería desechado para tales aplicaciones debido a su inestabilidad, siendo consiguientemente sustituido por otros. Actualmente, su uso más habitual en el campo de la conservación es como adhesivo, recubrimiento y consolidante de metales (Frigilene $^{\circledR}, \mathrm{HMG}$ ), para algunos de los cuales según ciertos autores ha demostrado tener una estabilidad aceptable, debido a los aditivos que se añade en estas formulaciones (Shashoua, Bradley y Daniels 1992), aunque estudios comparativos recientes de envejecimiento con otros productos acrílicos demuestran el incremento de su fragilidad (González y Leal 2008: 223-231). La inherente inestabilidad, del nitrato de celulosa, que proviene de la baja fortaleza de ciertos enlaces de su estructura química, y el procesado del material (Mills y White 1994: 164) son las causas de los procesos de degradación a través del tiempo de los objetos realizados con dicho producto. El material posee, por tanto, un tiempo de vida limitado que sólo puede alargarse tomando las medidas idóneas de conservación-restauración y controlando de forma precisa las condiciones ambientales en que se conservan las piezas hechas con esta sustancia. 


\section{Problemas de conservación de las peinetas}

\section{Estudio previo del estado de conservación}

En la actualidad, la colección de peinetas del Museo del Traje, CIPE presenta en general buen estado de conservación, sin que se observen deterioros significativos aparentes que pongan en peligro su integridad: conservan su unidad matérica, estructural y estética original, aunque presentan ligeros daños como erosiones superficiales o pérdidas de elementos, casi siempre relacionados con el uso que tuvo la pieza antes de su entrada en el Museo. No obstante, los controles periódicos efectuados pusieron de manifiesto el progresivo deterioro experimentado por una serie de peinetas fabricadas con un material que en un primer momento se identificó como un polímero plástico de naturaleza desconocida. El conjunto afectado tenía, además, otra característica común, ya que se trata de piezas fechadas entre 1910 y 1940, lo que hacía suponer que estábamos ante un polímero artificial sintetizado a partir de la celulosa. En 2008, la degradación de alguna de ellas era tan severa que incluso se había extendido a elementos como la etiqueta identificativa y el envoltorio, ajenos a la pieza aunque obviamente muy próximos físicamente a ella. La alteración, entendida como la percepción sensorial de los fenómenos de degradación, era, pues, evidente.

En estos casos de extrema degradación, se observaron diversos grados y tipos de alteraciones. Entre las piezas que presentan mayor grado de deterioro destacan tres, identificadas con los números de inventario CE012434, CE012440 Y CE064687. Su grado de deterioro actual es similar, han perdido su integridad e identidad material, quedando prácticamente reducidas a un estado de "ruina"; son irrecuperables su morfología y su estética, por conservarse en forma de múltiples fragmentos frágiles, aunque felizmente, en los tres casos, existen porciones de mayor tamaño que nos dejan ver parte de los motivos decorativos incisos o aplicados (aplicaciones de vidrio). Los fragmentos que corresponden a la parte superior de la peineta, en los que el material presenta una mayor consistencia, testimonian el trabajo artesano-industrial de la época. Salvo estos restos que conservan parte de su lectura, el resto de la peineta, tiene múltiples cuarteados de aristas limpias y profundas con un esquema repetitivo de formas geométricas cuadrangulares. Este tipo de alteración física se debe a procesos químicos de deterioro inherentes a la naturaleza material. Otro tipo de deterioro detectado en las peinetas consiste en la transformación del color, brillo y transparencia originales. Los procesos de fotooxidación producen amarilleamiento y decoloración. Algunas peinetas incoloras, han pasado a tonalidades verde-amarillentas, mientras que otras han tornado hacia tonos pardos. Una última alteración observada es que de forma generalizada la superficie es pegajosa al tacto. Una posible explicación es el incremento superficial de la concentración de moléculas de pequeño tamaño empleadas como plastificantes, ya que a la vez las piezas desprenden fuerte olor a alcanfor, signo inequívoco del progresivo y actual deterioro que está sufriendo el material constitutivo.

\section{Causas de degradación}

Una primera hipótesis es el origen químico de las posibles causas o factores que han contribuido a desencadenar los procesos de deterioro sufridos por las peinetas, debido a la naturaleza inestable propia del material.

De ahí que la primera medida adoptada fuera, además de extremar el control de unos procesos que se antojaban plenamente activos, identificar con precisión el tipo de material plástico constituyente de las peinetas más afectadas, para lo cual se recurrió a los especialistas del Laboratorio de Análisis de Materiales del Instituto del Patrimonio Conservación de España. 
Elena Gómez Sánchez, María Antonia Herradón Figueroa, Silvia Montero Redondo y María Antonia García
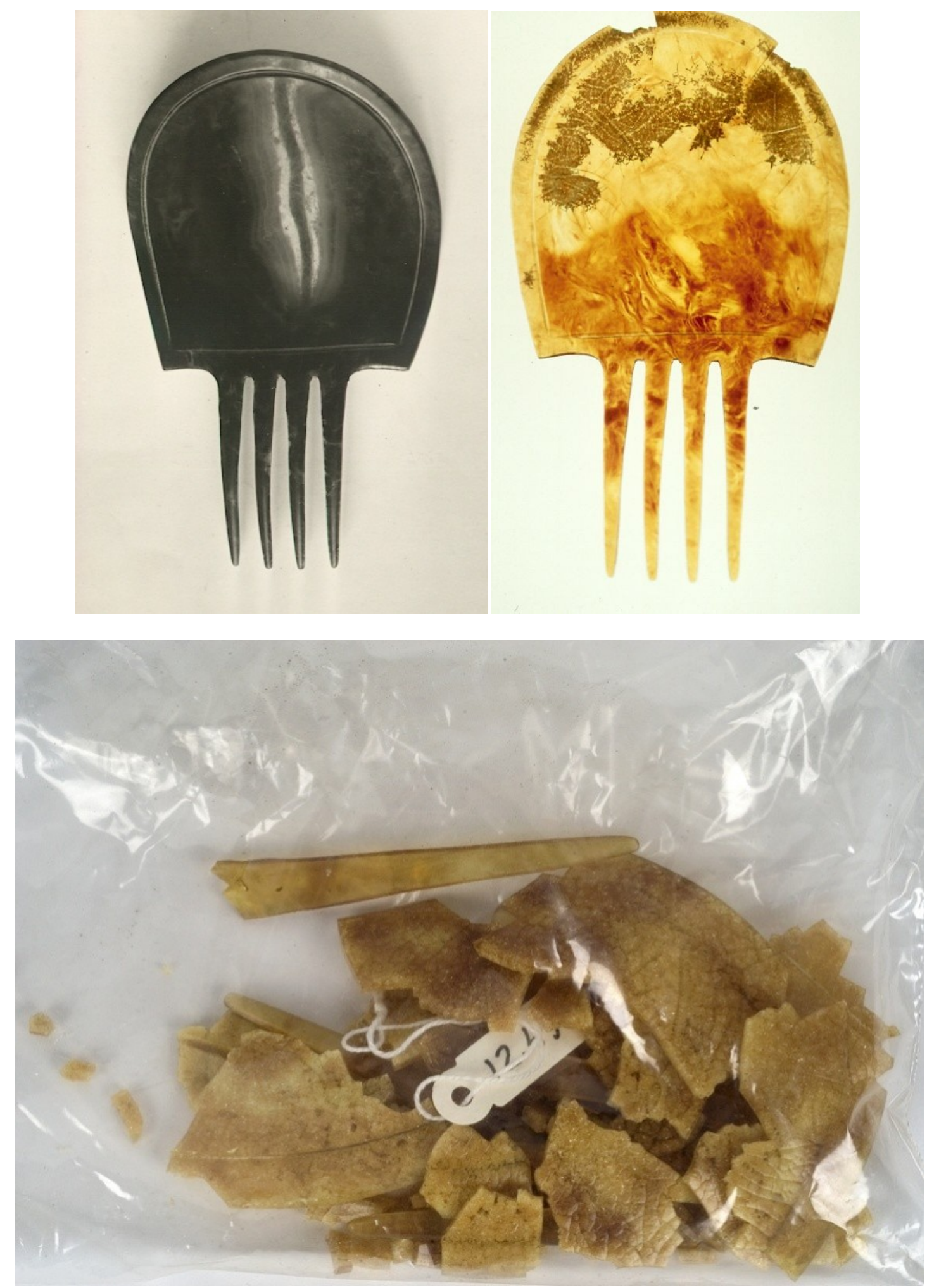

Figura 1: a) Peineta CE012434 en 1949, año de su ingreso en el Museo; b) La misma peineta con un deterioro muy avanzado en 1995; c) La misma peineta, ya completamente deteriorada, en 2008. 
Mientras que los procesos primarios de degradación del nitrato de celulosa parecen ser lentos (Selwitz op. cit.; 15) y esto hace que exista un primer período de cierta estabilidad, los procesos secundarios, consecuencia de los productos generados en los procesos primarios de degradación, son altamente destructivos. Los modos fundamentales de degradación primaria son degradación química, fotoquímica y térmica. En las condiciones lumínicas (ausencia de radiación UV lejana) y de temperatura más habituales (temperatura ambiente), los mecanismos más comunes de degradación son los siguientes:

- Degradación química. Se debe principalmente a la hidrólisis autocatalítica de los grupos nitro de la cadena, y la consecuente liberación de ácido nítrico $\left(\mathrm{HNO}_{3}\right)$ y otros gases que catalizan a su vez la degradación (Mills y White 1994) unido a la presencia de trazas del ácido sulfúrico $\left(\mathrm{H}_{2} \mathrm{SO}_{4}\right)$ utilizado en la fabricación del nitrato de celulosa y no eliminado completamente y la humedad, debida a la higroscopicidad de este último ácido. El proceso de alteración es inherente al material y no puede ser suprimido, aunque sí puede ser ralentizado a bajas temperaturas. El ácido nítrico generado en este proceso es el causante de la degradación de los materiales contiguos a las peinetas de nitrato de celulosa (tales como el papel y envoltorios plásticos tales como Mylar ${ }^{\circledR}$ o Melinex ${ }^{\circledR}$, que se degradan en presencia de ácidos muy fuertes). La acidez puede ser detectada con tiras de $\mathrm{pH}$. También se puede dar la hidrólisis básica, cuando estén presentes pigmentos básicos capaces de reaccionar con el nitrato de celulosa.

- Degradación fotoquímica. Implica la ruptura de los ésteres de nitrato por radiación con luz visible y del UV cercano, y tiene las mismas consecuencias que la degradación química.

- Degradación física. La pérdida del alcanfor en materiales donde se ha usado como plastificante y estabilizante, debido a su relativamente alta presión de vapor [Physical Data Handbook], vuelve el objeto frágil y hace que presente grietas e incluso roturas.
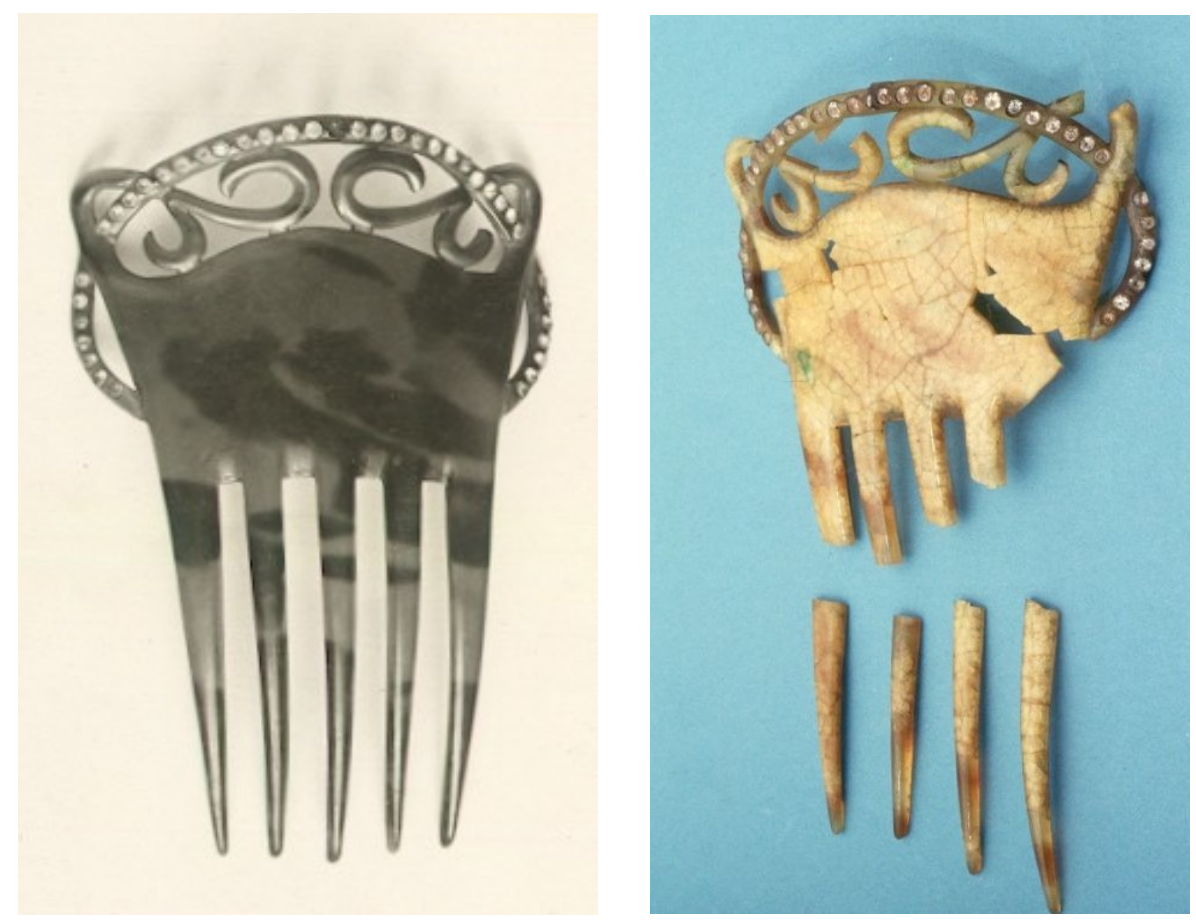

Figura 2: a) Peineta CE012440 en 1949, año de su ingreso en el Museo; b) La misma peineta en 1995, con un deterioro muy avanzado. 


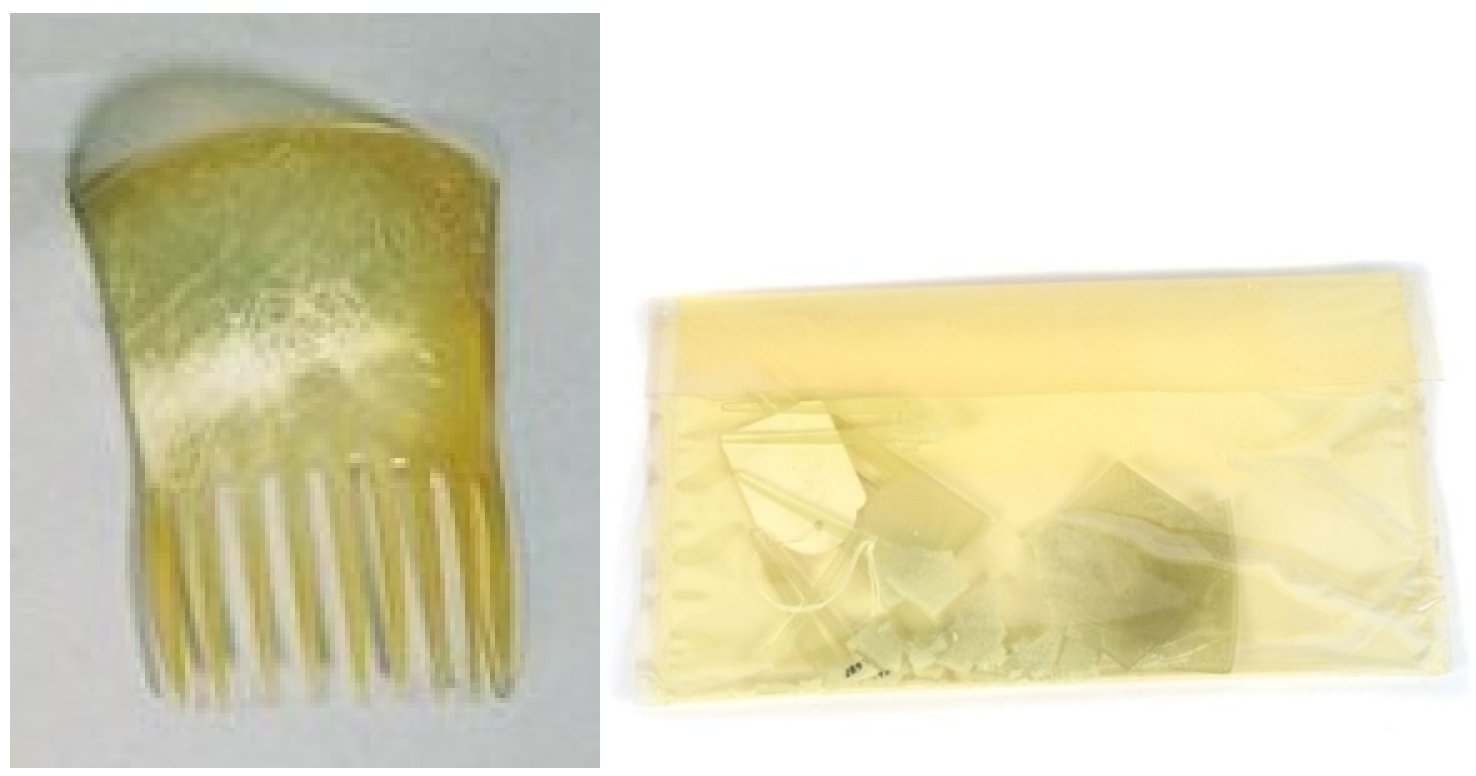

Figura 3: a) Peineta CE064687 en 2001, año de su ingreso en el Museo. B) Estado de conservación de la misma peineta en 2008.

\section{Análisis de piezas. Objetivos materiales y métodos y resultados.}

Ya hemos mencionado que los análisis de las peinetas fueron solicitados por el Museo del Traje al Instituto del Patrimonio Cultural de España, para determinar las causas de la evidente degradación que presentaban. Esto hizo que los objetivos de los análisis se centraran en los siguientes puntos:

- Identificar el material constitutivo de las peinetas y del aditivo usado como plastificante, así como establecer su grado de deterioro.

- Determinar la composición de los materiales usados como envoltorios de las peinetas, tanto aquellos que muestran degradación, como aquellos que se conservan íntegros a pesar de la acidez detectada en las tiras indicadoras. En algunos casos, el papel $\mathrm{pH}$ indicaba un valor en torno a 2.5 , suficiente para degradar las etiquetas identificativas.

- Recomendar las condiciones más idóneas de conservación.

Las muestras analizadas de las peinetas y los envoltorios según se indica en la tabla 1 se estudiaron por espectrometría molecular. Las micromuestras extraídas de las peinetas fueron dispersadas en pastillas de $\mathrm{KBr}$ y posteriormente analizadas por espectroscopia de infrarrojos por transformada de Fourier (FTIR) en bancada (Bruker-Equinox 55). Los materiales de los envoltorios se estudiaron por micro-FTIR con un objetivo ATR, directamente sobre la superficie de las muestras de plásticos contenedores, sin tratamiento previo.

La exposición de los resultados de los análisis sigue el orden establecido en los objetivos mencionados anteriormente.

El primer objetivo se centraba por tanto en estudiar la composición de las peinetas. El material del que están compuestas las tres peinetas analizadas (CE012434, CE012440, CE064687) es nitrato de celulosa. La identificación del material constitutivo de las piezas se hizo por comparación de los espectros de FTIR de muestras de las distintas peinetas con el de muestras "patrón" conocidas de 
nitrato de celulosa. Así, se pudo distinguir bandas sobre 1650 y 1283 (indicativa de enlaces N-O y C-O como los presentes en el NC), 1069 (ídem C-O), 2960 (C-H) y 844 cm$^{-1}$ (N-O).

También se ha podido detectar la presencia de alcanfor como plastificante en estos materiales, pudiendo existir otros aditivos no identificados. Se ha localizado la frecuencia típica de este producto en el espectro de FTIR $\left(\sim 1735 \mathrm{~cm}^{-1}\right)$. Además, en dos de las muestras (MTP1, de la peineta 12434, y M'TP3, de la peineta 64687) era evidente el olor típico de esta sustancia.

Tabla 1. Muestras analizadas

\begin{tabular}{|c|c|c|c|}
\hline Peineta & Comentarios & Muestra & Descripción de la muestra \\
\hline \multirow{3}{*}{12434} & \multirow{3}{*}{$\begin{array}{l}\text { Peineta completamente fragmentada, } \\
\text { guardada en una bolsa abierta de } \\
\text { plástico fina e incolora }\end{array}$} & MTP1A, MTP1A-INT & Fragmento traslúcido de púa (interior) \\
\hline & & MTP1B,MTP1B-EXT & Cuarteado (exterior) \\
\hline & & MTP1C & Envoltorio plástico \\
\hline \multirow{3}{*}{12440} & \multirow{3}{*}{$\begin{array}{l}\text { Peineta fragmentada, almacenada en } \\
\text { una caja de cartón }\end{array}$} & MTP2i & Adhesivo de unión de una grieta \\
\hline & & MTP2ii, MTP2ii-EXT & Cuarteado (exterior) \\
\hline & & MTP2iii-INT & Fragmento traslúcido de púa \\
\hline \multirow{3}{*}{64687} & \multirow{3}{*}{$\begin{array}{l}\text { Peineta fragmentada que desprende } \\
\text { fuerte olor a alcanfor, embalada en } \\
\text { un envoltorio de plástico duro con } \\
\text { coloración amarillenta }\end{array}$} & MTP3A,MTP3A-EXT & Cuarteado \\
\hline & & MTP3B, MTP3B-INT & Fragmento translúcido púa \\
\hline & & MTP3D & Envoltorio (plástico duro amarillento) \\
\hline 64693 & & MTP4 & Envoltorio (plástico duro amarillento) \\
\hline 64698 & & MTP5 & Envoltorio (plástico duro amarillento) \\
\hline 64688 & & MTP6 & Envoltorio (plástico duro amarillento) \\
\hline 64694 & & MTP7 & Envoltorio (plástico duro amarillento) \\
\hline
\end{tabular}

Para evaluar el alcance de la degradación se han comparado los espectros de FTIR de dos tipos de muestra tomadas para cada peineta. Se ha distinguido entre material craquelado o completamente fragmentado, tomando una muestra del exterior de los fragmentos, y material traslúcido, sin 
degradación aparente (generalmente las púas). En el segundo caso, y para asegurar una medida representativa, se ha extraído la muestra del interior de estos fragmentos.

En la columna central de la tabla 2 se advierte una disminución de la intensidad de la señal característica del alcanfor $\left(\mathrm{C}=\mathrm{O}, 1735 \mathrm{~cm}^{-1}\right)$ con respecto a una de las señales características del nitrato de celulosa $\left(\mathrm{C}-\mathrm{O}, 1280 \mathrm{~cm}^{-1}\right)$ al comparar las muestras menos degradadas (recuadros sombreados de la tabla) con las craqueladas (recuadros no sombreados). Se observa asimismo en la columna derecha de la tabla el aumento de la intensidad de la señal a $1380 \mathrm{~cm}^{-1}$ en las muestras cuarteadas con respecto a la señal de referencia del NC $\left(1280 \mathrm{~cm}^{-1}\right)$, aunque esta última relación no supone un parámetro de evaluación de la degradación tan válido como el anterior ya que no es consistente en todas las muestras.

El segundo objetivo se refería al análisis de los materiales usados como envoltorios de las peinetas y esto motivó que se hayan elegido como muestras representativas: MTP1C (fragmento del plástico utilizado para envolver la peineta 12434) y MTP4 (idem peineta CE064693), por las razones que se indican a continuación. En el primer caso, por tratarse de un plástico fino que no presentaba degradación aparente y de un tipo que no había sido utilizado para contener ninguna otra peineta, y en el caso de la muestra MTP4 por representar igualmente otros plásticos contenedores (MTP5-7), en lo que respecta a la naturaleza del material y a su aspecto (degradación en forma de coloración amarillenta).

El análisis por ATR ha revelado que la muestra MPT1C corresponde a un polietileno (Derrick, Stulik y Landry 1999: 110), mientras que la muestra MTP4 se identificó como tereftalato de polietileno por comparación de su espectro de infrarrojo con el de una muestra de Mylar®.

Tabla 2

\begin{tabular}{|c|c|c|}
\hline Muestra & $\begin{array}{l}\text { Proporción de Intensidad } \\
\left(1735 \mathrm{~cm}^{-1}\right) /\left(1280 \mathrm{~cm}^{-1}\right)\end{array}$ & $\begin{array}{l}\text { Proporción de Intensidad } \\
\left(1380 \mathrm{~cm}^{-1}\right) /\left(1280 \mathrm{~cm}^{-1}\right)\end{array}$ \\
\hline MTP1B-EXT (craquelado) & 0.47 & 0.52 \\
\hline MTP1A-INT (traslúcido) & 0.60 & 0.51 \\
\hline MTP2ii-EXT (craquelado) & 0.41 & 0.35 \\
\hline MTP2iii-(INT) (traslúcido) & 0.53 & 0.34 \\
\hline MTP3A-EXT (craquelado) & 0.65 & 0.58 \\
\hline MTP3B-INT (traslúcido) & 0.70 & 0.64 \\
\hline
\end{tabular}

Uno de los mecanismos de degradación, previamente expuesto, se basa en la pérdida del plastificante (alcanfor) por sublimación, lo cual puede ocurrir a temperatura ambiente. Estudios 
previamente publicados (Derrick, Stulik y Ordonez 1993: 169-182) han hallado una correlación directa entre la falta de alcanfor de muestras de estos materiales y la degradación paulatina de las piezas. Por tanto, se ha procedido a verificar que en efecto existe pérdida de alcanfor en las muestras tomadas.

El Mylar ${ }^{\circledR}$ y el Melinex ${ }^{\circledR}$ son materiales inertes frente a la mayoría de disolventes orgánicos a temperatura ambiente, pero son hidrolizados en presencia de ácidos fuertes, tales como los vapores de ácido nítrico que segrega el nitrato de celulosa durante su proceso de degradación. Por ello, no son materiales idóneos para contener objetos donde esta sustancia esté presente.

\section{Pautas a seguir en la conservación preventiva de la colección de peinetas}

Como se ha comentado con anterioridad, los procesos de degradación del nitrato de celulosa son inherentes al propio material, e irreversibles. Aunque no pueden ser completamente eliminados, sin embargo, pueden ser ralentizados mediante el control de las condiciones ambientales en que se mantienen las piezas fabricadas de este material.

Una vez que hemos identificado el compuesto como nitrato de celulosa (olor a alcanfor, espectro de ATR de la superficie sin toma de muestra o de FTIR de una muestra) o como acetato de celulosa [olor a 'vinagre' (ácido acético), espectros de ATR o FTIR], se recomienda su aislamiento del resto de la colección. Los productos generados durante su degradación acelerarán la de los objetos cercanos sensibles. Aconsejamos también evitar su lavado con disoluciones acuosas, alcohólicas o acetona, en los que el NC es parcialmente soluble (Reilly 1991: 156) y el uso de productos de limpieza alcalino, que pueden provocar reacciones exotérmicas en presencia de ácido (Reilly 1991 :158).

Todas estas medidas de conservación serán más eficaces cuanto más temprana sea la detección del inicio de las alteraciones. La revisión regular de las colecciones en el caso de estos materiales, por tanto, fundamental. Signos de degradación son, entre otros, amarilleo, cambios de color y detección de acidez en los indicadores de $\mathrm{pH}$. La detección de este aumento de la acidez significa que las piezas cercanas se encuentran también en peligro, porque los vapores ácidos de la pieza más degradada acelerarán su descomposición aún cuando ésta no hubiera comenzado todavía. La aparición de pequeñas grietas, que con facilidad se pueden -erróneamente- achacar a fracturas mecánicas, marca el inicio de la fase final de alteración del material. Como resultado de esta fase, la pieza se fragmenta en pequeños trozos.

Las condiciones ambientales más deseables para piezas compuestas de nitrato de celulosa se resumen a continuación:

- Baja temperatura (Reilly 1991: 159).

- Tanto las piezas fabricadas en nitrato como en acetato de celulosa deben mantenerse a una baja humedad relativa (idealmente por debajo del $40 \%$ ), ya que la humedad es un factor que afecta a su estabilidad (Reilly 1991: 158).

- Deben almacenarse en envoltorios abiertos por dos lados, con el fin de facilitar la ventilación de los materiales tóxicos.

- Buena ventilación, para evitar la concentración de vapores ácidos, y disponer en las proximidades de los objetos (pero no en contacto con ellos) sustancias capaces de absorber vapores ácidos, tales como el carbonato de calcio. 
- Hay que evitar también poner en contacto estas piezas con materiales metálicos, ya que ciertos metales (hierro entre ellos) aceleran su degradación. Ello implica, además, aislar los aderezos indumentarios que contengan en su decoración pequeñas piezas metálicas.

- Aislamiento de otros materiales celulósicos.

- Contenedores resistentes a los ácidos fuertes, no metálicos e idealmente capaces de filtrar la luz ultravioleta.

- Proteger las piezas de luz UV, que acelera los procesos de degradación.

Las condiciones ideales para conservar estos accesorios sería introducirlos en el interior de una cámara a baja temperatura, a baja humedad relativa, y bajo ventilación. El almacenamiento de ciertas cantidades de alcanfor en la misma cámara, garantizando una atmósfera saturada de esta sustancia, impediría que el existente en las piezas sublimara, lo cual favorecería el comienzo de los procesos de degradación.

Siguiendo el máximo número de estas medidas se puede alargar notablemente la vida de piezas de NC. En caso contrario, la degradación de estos materiales es muy rápida una vez que se encuentra en etapas intermedias, y como hemos visto conlleva, finalmente, la fragmentación de las piezas y su destrucción.

Asimismo, el conocimiento de las características de fabricación y de los elementos que componen los materiales de objetos de nueva adquisición de la colección será fundamental para mejorar su futura conservación.

\section{Medidas de conservación preventiva. De la teoría del laboratorio a la práctica del museo.}

Tras estudiar e identificar los materiales constituyentes de las peinetas, los procesos de degradación y las posibles causas que han podido intervenir en su deterioro, hemos diseñado y puesto en práctica un protocolo de actuación que permita y asegure la correcta conservación de la colección, eliminando los factores de deterioro existentes hasta el momento y reduciendo el riesgo observado en la actualidad en su lugar de almacenamiento. Este protocolo de actuación en materia de conservación lo resumimos en 5 puntos que a continuación explicamos:

\section{Identificación de cada pieza.}

Cada pieza debe ser estudiada en profundidad desde el punto histórico-artístico, con datación cronológica, análisis de materias y técnicas de fabricación, con una exhaustiva documentación fotográfica que permita su estudio y control rutinario posterior, en cuanto a su conservación se refiere. Deben ir correctamente sigladas, con su número de inventario correspondiente, mediante métodos y materias inertes e inocuas para cada pieza en particular evitando el uso de métodos irreversibles y tintas con $\mathrm{pH}$ ácido.

\section{Clasificación (materias, estado de conservación).}

En primer lugar, ante los riesgos detectados por la interacción de sustancias emitidas por los materiales en forma de productos de degradación, establecemos una nueva clasificación de la colección de peinetas y procedemos a su almacenamiento en función de su naturaleza y estado de conservación. Así, las clasificamos y agrupamos una vez identificada la presencia de materiales inorgánicos como los metales u orgánicos como el carey, hueso, nitrato de celulosa, acetato de celulosa o cualquier tipo de material artificial o sintético de origen orgánico. Por tanto, diremos que 
el sistema de trabajo interdisciplinar establecido en este artículo, basado en el análisis e identificación del material constituyente de las peinetas de nitrato de celulosa, debe tener un seguimiento en el tiempo y ser extrapolado al resto de la colección.

Sin embargo, somos conscientes de que muchas de las peinetas (y la mayoría de los objetos depositados en los Museos) están realizadas con materiales muy diversos de distinta naturaleza que, irremisiblemente, conforman la pieza sin que sea posible su separación para garantizar su conservación. En este caso, nos vemos en la obligación de asumir que la intervención por parte de los profesionales implicados en la preservación de las colecciones, tiene un límite y que la vida de los materiales que conforman los bienes culturales, en ocasiones, tienen una "esperanza de vida" limitada.

\section{Restauración: intervenciones curativas.}

Las peinetas, una vez clasificadas son restauradas siguiendo los criterios de restauración establecidos y aceptados internacionalmente. Con ello, mediante limpiezas mecánicas eliminaremos los productos de degradación de su superficie. Posteriormente procederemos a su consolidación matérica mediante métodos y productos estables y eficaces que no presenten incompatibilidades, que sean fácilmente eliminados en superficie y que puedan ser sustituidos por otros en el futuro si dejan de cumplir su función. En el caso de las peinetas analizadas, somos conscientes de su irremediable pérdida puesto que los restos carecen de unidad potencial en sí mismos, al verse afectada su materia hasta un punto irrecuperable.

4. Diseño de soportes de conservación para almacén y transporte.

Una vez clasificadas y restauradas, hemos elaborado un soporte de conservación que garantiza su correcta conservación en las salas de reserva del actual museo en las que está depositada la colección. Al mismo tiempo, este tipo de soporte de almacenaje, cumple con los requisitos necesarios para transportar con seguridad las piezas.

Se trata de una caja de cartón de pH neutro, con una carga del 3\% de carbonato cálcico, es decir, una reserva alcalina capaz de absorber y neutralizar los vapores ácidos emitidos por las piezas de nitrato de celulosa. Está perforada en sus extremos para permitir la ventilación de los objetos del interior, sin que se cree una cámara estanca con la consecuente acumulación de vapores ácidos.

En su interior se disponen, en bandejas individuales, las peinetas organizadas previamente en función de su materia y estado de conservación. Cada una de ellas descansa sobre una base o lecho de muletón de algodón de $\mathrm{pH}$ neutro, que se adapta a la morfología de cada peineta. Este soporte permite que la superficie total de la pieza descanse y evita que se creen puntos de tensión diferencial. Para evitar la excesiva e innecesaria manipulación de las piezas que puede resultar perjudicial para su conservación, cada una permanece envuelta en papel de seda de $\mathrm{pH}$ neutro y cinta de algodón descrudada.

El siglado de las piezas garantiza su correcta identificación tanto de forma individual por medio de una etiqueta colgante de papel y tinta de $\mathrm{pH}$ neutro, como en grupo ya que cada caja informa de su contenido por medio de la relación de números de inventario de las piezas y de las correspondientes fotografías.

Por último, es necesario señalar que este sistema de almacenaje es perfectamente compatible con el resto de materiales que forman la colección. 

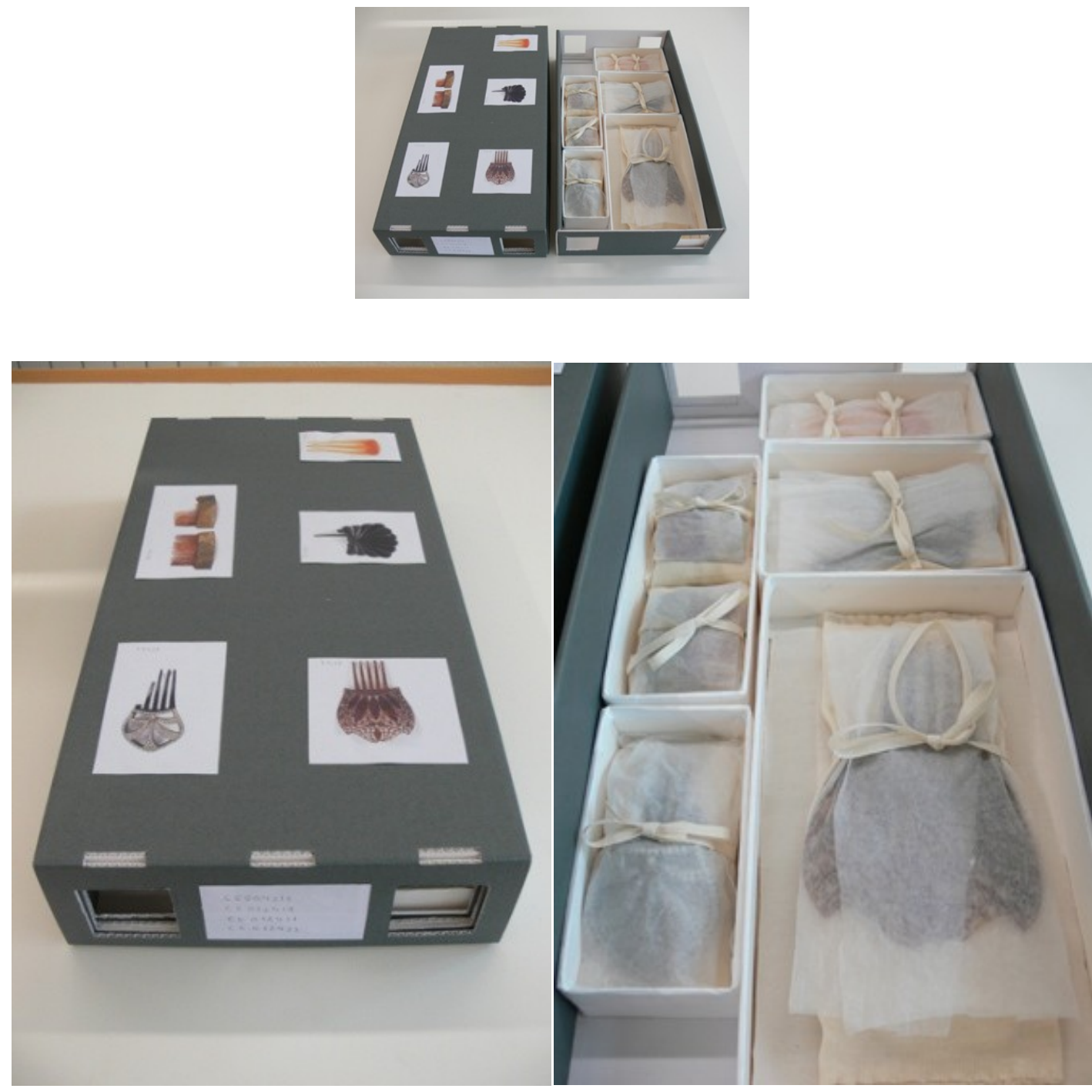

Figura 4: a) Soporte de almacenaje diseñado para la conservación de peinetas de NC; b) Interior de la misma con su tapa; c) Distribución en bandejas independientes.

\section{Medidas de control ambiental.}

Aplicando los protocolos de evaluación de riesgos sobre conservación de materiales plásticos de naturaleza orgánica desarrollados en el Instituto Canadiense de Conservación, podemos establecer que unas condiciones de conservación ambientales idóneas para la conservación de las peinetas de NC consisten en mantener unos valores ambientales de $15^{\circ} \mathrm{C}$ de Temperatura $(\mathrm{T})$ y de un $40 \%$ de Humedad Relativa (HR). Sin embargo, somos conscientes de que unos valores inferiores de y del orden de $10^{\circ} \mathrm{C}(\mathrm{T})$ y $30 \%$ de HR serían los más aconsejables para garantizar su pervivencia en los próximos cien años. Pero llegados a este punto, hemos de tener en cuenta también los medios reales con los que contamos en la actualidad en las salas de reserva del Museo del Traje que, puesto que se caracterizan por su estabilidad ambiental, son los ideales para conservar el resto de las colecciones que custodia el Museo del Traje, CIPE. Estamos convencidos, no obstante, que con motivo del próximo traslado de la colección de peinetas a su futura -y esperemos que definitivasede en el Museo Nacional de Etnografía de Teruel, se pongan en práctica estas recomendaciones para conservar los materiales poliméricos en las mejores condiciones posibles. De esta manera se garantizará su transmisión a las generaciones futuras. 
Por último diremos que, además de la revisión periódica de la colección, es necesario e imprescindible su análisis constante desde distintos puntos de vista. Una tarea que directamente compete e implica a todo personal técnico del museo -conservador de Museos y conservadorrestaurador de Bienes Culturales-, que son, en definitiva, los directos responsables de su cuidado y preservación.

\section{Bibliografía}

ALBUS, S.; BONTEN, C.; KESSLER, K.; ROSSI, G.; Wessel, T. (2007). Plastic Art - A Precarious Success Story“, Hamburg: AXA Art.

BOLLÉ, R.(2004). Le peigne dans le monde, Paris: Éditions Hoëbeke.

BRENNER, R. (1999). Celluloid: Collectibles from the Dawn of Plastics, Atglen: Schiffer Publishing.

DERRICK, M. R.; STULIK, D.C.; LANDRY, J. M. (1999). Infrared Spectroscopy in Conservation Science, Los Angeles: The Getty Conservation Institute.

DERRICK, M. R. ; STULIK, D. C.; ORDONEZ, E. (1993). "Deterioration of cellulose nitrate sculptures made by Gabo and Persner" en Saving the Twentieth Century: The Conservation of Modern Materials, Canada: Canadian Conservation Institute: 169-82.

DOMENICONI, M. (1992). Pettini ornamentali. Ornamental Hair-Combs, Milano: BE-Ma Editrice.

HAGUE, N. (1985). Combs and Hair Accessories, Cincinnati: Seven Hills Books.

ESPINOZA MORAGA F. ARAYA MONASTERIO, C. (2006). "Materiales modernos en la colección textil del Museo Histórico Nacional", Conserva, 10: 55-70.

GONZÁLEZ, E., LEAL, J. con la colaboración de GÓMEZ, M., GARCÍA, E., RUIZ., P. Y NAVARRO, P. (2008). "Comportamiento de consolidantes de metales sometidos a envejecimiento acelerado", Bienes Culturales. Revista del Instituto del Patrimonio Cultural Español no 8: 223-231.

LAVÉDRINE, B. (2003). A Guide to the Preventive Conservation of Photograph Collections, Los Angeles: Getty Publications.

MICHALSKI, S. (2009). Manual de Control de Riesgos en las Colecciones, Ontario: Instituto Canadiense de Conservación.

MILLS, J. S., WHITE, R. (1994). The Organic Chemistry of Museum Objects, Oxford: Butterworth-Heinemann.

REILLY, J. A. (1991). "Celluloid objects: their Chemistry and Preservation", Journal of the American Institute for Conservation, 30: 145-162.

SHASHOUA, Y., BRADLEY, S. M., DANIELS, V. D. (1992). "Degradation of Cellulose Nitrate Adhesive", Studies in Conservation, 37, 113-119.

SELWITZ, C. (1988). Cellulose Nitrate in Conservation, Marina del Rey: Getty Conservation Institute. 


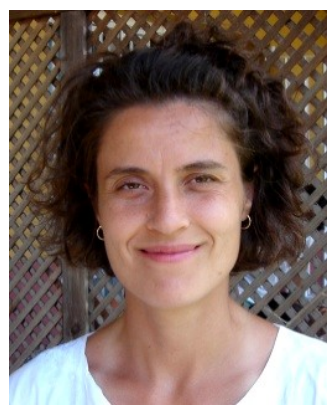

\section{A. García Rodríguez}

Instituto del Patrimonio Cultural de España

c/ Pintor el Greco 4, 28040, Madrid

antonia.garcia.r@mcu.es

María Antonia García Rodríguez, licenciada en Ciencias Químicas (UCM). Entre 2001 y 2005 trabajó como asistencia técnica en el estudio de técnicas instrumentales aplicadas a la Investigación y Documentación del Patrimonio Histórico en el IPCE, donde actualmente, desde 2006, está como Titulado Superior.

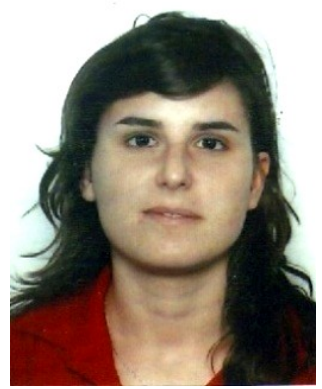

\section{E. Gómez Sánchez}

Laboratorio de Investigación Rathgen, Museos Nacionales de Berlín Rathgen-Forschungslabor, Staatliche Museen zu Berlin, Schlossstr. 1A 14059 Berlín, Alemania e.gomez@smb.spk-berlin.de

Elena Gómez Sánchez, licenciada en Química Orgánica (UCM, 2003), recibió en 2008 su doctorado en Síntesis Orgánica (CSIC). Comenzó entonces su especialización en Ciencia de la Conservación bajo la dirección de Marian García y Marisa Gómez (IPCE). Actualmente está contratada como científico en el Laboratorio Rathgen (Museos Nacionales de Berlín).

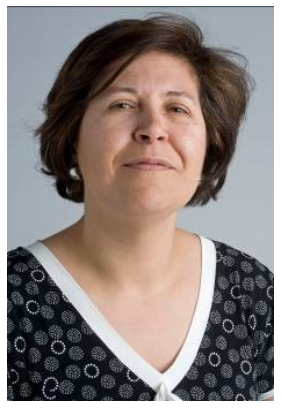

\section{A. Herradón Figueroa}

Museo del Traje, Centro de Investigación del Patrimonio Etnológico

Avda. Juan de Herrera, 2

28040 Madrid

mantonia.herradon@mcu.es

María Antonia Herradón Figueroa, licenciada en Historia Antigua y DEA en Historia del Arte (UCM, 2009), desde 1992 pertenece al Cuerpo Facultativo de Conservadores de Museos. Desde entonces ha trabajado como responsable, entre otras, de las colecciones de joyería, adorno personal y religiosidad. 


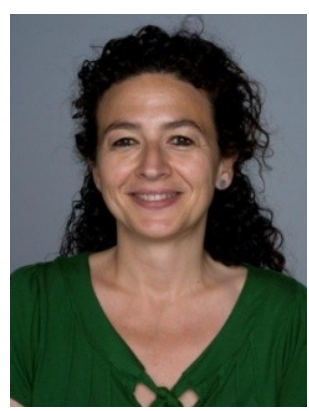

\section{S. Montero Redondo}

Museo del Traje, Centro de Investigación del Patrimonio Etonológico

Avda. Juan de Herrera, 2

28040 Madrid

silvia.montero@mcu.es

Silvia Montero Redondo, licenciada en Historia del Arte (UAM, 1992); Diplomada en Conservación y Restauración de BBCC, especialidad Pintura (ESCRBC, Madrid, 1997) y textiles. Actualmente es responsable de la conservación-restauración de la colección textil e indumentaria del Museo del Traje, CIPE. 